\title{
Modificación del índice de calidad de riberas: Inclusión del componente social en la evaluación de la calidad ribereña de la microcuenca del río Burío-Quebrada Seca
}

\author{
Riparian Forest Quality Index Modification: Inclusion of the Social Component in the \\ Assessment of the Riparian Quality of the Burio-Quebrada Seca River Micro-Watershed
}

Fabián Araya-Yannarella

fabian.araya.yannarella@una.cr

Escuela de Ciencias Biológicas

Universidad Nacional

Heredia, Costa Rica

Allan Fernández-Hernández

ajfernandez@uned.ac.cr

Escuela de Ciencias Exactas y Naturales

Universidad Nacional Estatal a Distancia

San José, Costa Rica

Recibido-Received: 10/dic/2015 / Corregido-Corrected: 22/may /2016.

Aceptado-Accepted: 5/set/2016 / Publicado-Published: 31/ene /2017.

\begin{abstract}
Resumen
Esta investigación se basó en la comparación de los resultados obtenidos mediante la aplicación del índice de calidad de riberas-QBR (Munné et al., 2003) y el índice de calidad de riberas modificado-QBRm (Araya y Fernández, 2011) en seis zonas de la microcuenca del río Burío y su tributario Quebrada Seca, ubicada en las provincias de Heredia y Alajuela, Costa Rica. El objetivo fue analizar el estado de la vegetación ribereña mediante la modificación del índice original (QBR) con la inclusión del componente social. Los resultados obtenidos de la aplicación del índice QBR fueron de 95 puntos ("muy buena" calidad) en los puntos de muestreo 1 y 2 (zona alta), 40 y 45 puntos ("mala" calidad) en los puntos de muestreo 3 y 4 (zona media), respectivamente y 15 y 10 puntos ("muy mala" calidad) en los puntos de muestreo 5 y 6 , respectivamente (zona baja). Los resultados obtenidos a partir de la aplicación del índice QBRm mantienen la misma tendencia y no presentaron diferencias estadísticamente significativas (IC 95\% p: 0, 092). El índice QBRm puede ser utilizado para complementar el componente estrictamente biológico del índice QBR y así contribuir a la toma de decisiones relacionadas con el manejo integral de la microcuenca.
\end{abstract}

Palabras claves: Conservación del agua; gestión de los recursos hídricos; cuenca de drenaje; vegetación; integración social.

\begin{abstract}
This research was based on the comparison of the results obtained by applying the Riparian Quality Index-QBR (Munné et al., 2003) and the Riparian Quality Index modified-QBRm (Araya y Fernández, 2011) in six areas of the Burío-Quebrada Seca river micro-watershed, located in the provinces of Alajuela and Heredia, Costa Rica. The objective was to analyze the status of riparian vegetation by modifying the original index (QBR) with the inclusion of the social component (QBRm). The results
\end{abstract}


UNICIENCIA Vol. 31, No. 1, pp. 39-49. Enero-junio, 2017.

ISSN Electrónico: 2215-3470

URL: www.revistas.una.ac.cr/uniciencia

DOI: $\underline{\text { http://dx.doi.org/10.15359/ru.31-1.5 }}$

Email: revistauniciencia@una.cr

of the application of the QBR index was 95 points ("very good" quality) at sampling points 1 and 2 (upper area of micro-watershed), 40 and 45 points ("bad" quality) at sampling points 3 and 4, respectively (middle area of micro-watershed), and 15 and 10 points ("very poor" quality) at the sampling points 5 and 6, respectively (lower area of micro-watershed). The results obtained from the applicPaation of QBRm index maintained the same trend and no statistically significant differences (CI 95\% p: 0, 092) appeared. The QBRm index can be used to complement the strictly biological component of the QBR index and to contribute with decisions related to the integral management of the micro-watershed.

Keywords: water conservation, water resources management; drainage basins; vegetation; social integration.

A lo largo de los márgenes del cauce de corrientes fluviales se encuentran franjas angostas de vegetación que pueden definirse como la interfase entre los ecosistemas acuáticos y terrestres (Burt et al., 2002; Granados, Hernández y López, 2006). A este tipo de comunidades vegetales que actúan como ecotonos se les conoce con el término de vegetación ribereña o riparia (Ceccon, 2003). Este tipo de vegetación se diferencia de la vegetación aledaña por poseer un mayor número de especies siempre verdes (Rzedowski, 1994 y Lamprecht, 1990, citados por Rodríguez, 2006) y porque sostienen ecosistemas estructuralmente más diversos y más productivos en biomasa animal y vegetal (Díaz y Rosales, 2006).

Estas comunidades vegetales están constituidas, en general, por especies pioneras de fácil reproducción y con tasas de crecimiento aceleradas. Su composición, estructura y disposición están determinadas principalmente por factores hidrológicos y fluviales, aunque también se ven influenciadas por procesos erosivos y de sedimentación, factores climáticos como la temperatura y la precipitación, el tipo de relieve y por las características del suelo (Huggenberger, Hoehn, Breschta y Woessner, 1998). La combinación de estos factores, aunada a los distintos usos del suelo que se desarrollan a lo largo de la cuenca, originan zonaciones longitudinales de vegetación, lo que genera diferencias entre las partes alta, media y baja. También se producen zonaciones o sucesiones de vegetación en un plano transversal, es decir, desde el centro del cauce del río hacia fuera o viceversa (en un eje de humedad), originando diferencias en la composición y estructura de las comunidades vegetales (González y García, 1998).

Las áreas ribereñas que presentan vegetación generan una variedad de servicios ecológicos en las cuencas y microcuencas, los cuales son considerados de gran importancia. Por ejemplo, estas comunidades vegetales sirven de filtro entre el río y los ambientes adyacentes, lo que impide o retarda el flujo de la escorrentía hacia el cauce, la cual en muchas ocasiones trae consigo agroquímicos, nutrientes (principalmente nitrógeno y fósforo) y productos orgánicos provenientes de las actividades antropogénicas y de los cambios en los usos del suelo (Burt et al., 2002; Carlyle y Hill, 2001; Dhondt, Boeckx, Verdehoest, Hofman y Van Cleemput, 2006; Granados et al., 2006). También, permite amortiguar los procesos de sedimentación de los lechos de los ríos y de erosión en las riberas (González y García, 1998; Pinay y Decamps, 1988; Timoney, Peterson y Wein, 1997), poseen una gran influencia sobre la calidad del agua y del ecosistema fluvial, debido a quela vegetación, principalmente la arbórea, sombrea el cauce del sistema acuático y contribuye al control del nivel de insolación y del régimen de temperaturas de las aguas; además, aumenta la cantidad de oxígeno disuelto, regula la entrada de luz al sistema y retarda el crecimiento de las algas (Ahola, 1990 y Dawson y Haslam, 1983, citados por González y García, 1998; Granados et al., 2006). 
La vegetación ribereña también aporta la materia orgánica que sirve como base de las cadenas tróficas del ecosistema acuático, principalmente como fuente de alimento de macro invertebrados y juega un papel importante en el manejo integrado de plagas en las zonas agrícolas aledañas, ya que se ha evidenciado, mediante estudios previos, que la avifauna que anida en esta zona es depredadora de roedores e insectos que atacan los cultivos (Ceccon, 2003; Granados et al., 2006).

Una de las funciones ecológicas más relevantes que poseen estas comunidades vegetales es su capacidad de actuar como corredor biológico, proporcionando a las distintas especies animales protección, sombra, zonas de descanso, de alimentación, de paso, migración y un microclima no tan fluctuante a lo largo del río (Ceccon, 2003; Granados et al., 2006; González y García, 1998). Estas especies de fauna silvestre, a su vez, son fundamentales en el corredor biológico, ya que dispersan las semillas y favorecen la regeneración natural de la vegetación ribereña (Gregory, Frederick, McKee y Cummins, 1991).

Cabe señalar que la vegetación ribereña no solo genera beneficios ecológicos, sino que también brinda una serie de servicios a los seres humanos que ayudan a mejorar su calidad de vida, por ejemplo, funciona como un agradable sistema paisajístico y provee especies vegetales que pueden ser utilizadas para fines forestales, comerciales y medicinales. Además, favorece la recarga de mantos acuíferos, esenciales para satisfacer las necesidades humanas (Sorensen, Barzetti, Keipi y Williams, 1998).

No obstante, en las últimas décadas, Costa Rica ha experimentado un fuerte deterioro de sus cuencas y microcuencas (principalmente las ubicadas en el Valle Central), debido a la presión ejercida por el modelo de desarrollo que prioriza el bienestar socioeconómico ante la conservación ambiental. Esta situación ha ocasionado un aumento en la contaminación de los cursos de agua, acuíferos y de las zonas ribereñas, principalmente, con desechos sólidos, lo cual ha provocado que estos bosques sean convertidos en relictos cada vez más pequeños, sobre todo en los límites urbanos (Zamora, 2002).

La microcuenca del río Burío-Quebrada Seca presenta una altísima vulnerabilidad ambiental, evidenciada en la degradación de sus riberas, en la contaminación de sus aguas y en la desprotección de nacientes, entre otras. El impacto directo e indirecto de las actividades antropogénicas que se desarrollan en esta microcuenca pone en peligro el bienestar de sus pobladores y del ambiente. Las consecuencias del deterioro son evidentes desde años atrás, sin embargo, eventos cada vez más dramáticos demuestran que las condiciones, lejos de mejorar, empeoran. Las comunidades donde los ecosistemas ribereños han desaparecido o se reducen a pequeños sectores con vegetación alterada, escasa e inestable son propensas a inundaciones, especialmente las ubicadas en la parte media y baja de la microcuenca. Las inundaciones del 2007 afectaron no solo los cantones de Flores y de Belén, sino que produjeron fuertes daños en partes más altas, como en los cantones de Heredia, Barva y San Rafael. Con ello se evidencia el aumento del deterioro de la microcuenca (Coto, Benavides y Salgado, 2008; Oviedo 2007; Zamora, 2002).

Por todo lo mencionado anteriormente, por el creciente desarrollo de la infraestructura y la pobre planificación urbana de los cantones del país, estudiar y monitorear la calidad de la vegetación de las riberas de los ríos es imprescindible. Precisamente, una de las herramientas para verificar la calidad de las riberas es el índice de calidad de riberas (QBR), el cual se originó a partir de estudios realizados en España y ha sido aplicado en diferentes ríos de características similares en los continentes europeo y americano (Aguilella, Riera, Gómez-Serrano, Mayoral, Moreyra y 
L. Botànic, 2005; Fernández, Rau y Arriagada, 2009; Gutiérrez y Alonso, 2000; Palma, Figueroa y Ruiz, 2009; Rodríguez-Téllez, Domínguez-Calleros, Pompa-García, Quiroz-Arratia y Pérez López, 2012; Suárez et al., 2002). Básicamente, consiste en una hoja de datos que se completa de manera sencilla en el campo, de acuerdo con las características tanto de la vegetación ribereña observada como del canal fluvial (Munné, Prat, Solá, Bonada y Rieradevall, 2003).

Tomando en cuenta la diferencia entre las características de los ríos europeos con los costarricenses, aunado al hecho de que al aplicar el índice QBR el resultado que se obtiene es basado en características biológicas y estructurales sin tomar en cuenta el componente social, se propuso una modificación al índice para integrar la opinión de las personas relacionadas directamente con la microcuenca y analizar si estas modificaciones provocan un cambio en los resultados finales cuando se aplican ambos índices. Esto, con la finalidad de mantener un solo instrumento de evaluación, sencillo de llenar y que involucre no solo la parte biológica sino también la parte social.

\section{Metodología}

El estudio se realizó en la microcuenca del río Burío-Quebrada Seca. El río Burío nace en Los Ángeles de San Rafael de Heredia y recorre parte del cantón de Barva; en su zona media atraviesa el cantón Central de Heredia y en el límite de este con el cantón de Flores se une a su tributario Quebrada Seca. Estos continúan su recorrido (como río Burío-Quebrada Seca) por los cantones de Flores y Belén, para finalmente desembocar en el río Bermúdez, en San Rafael de Alajuela (Zamora, 2002) (Figura1).

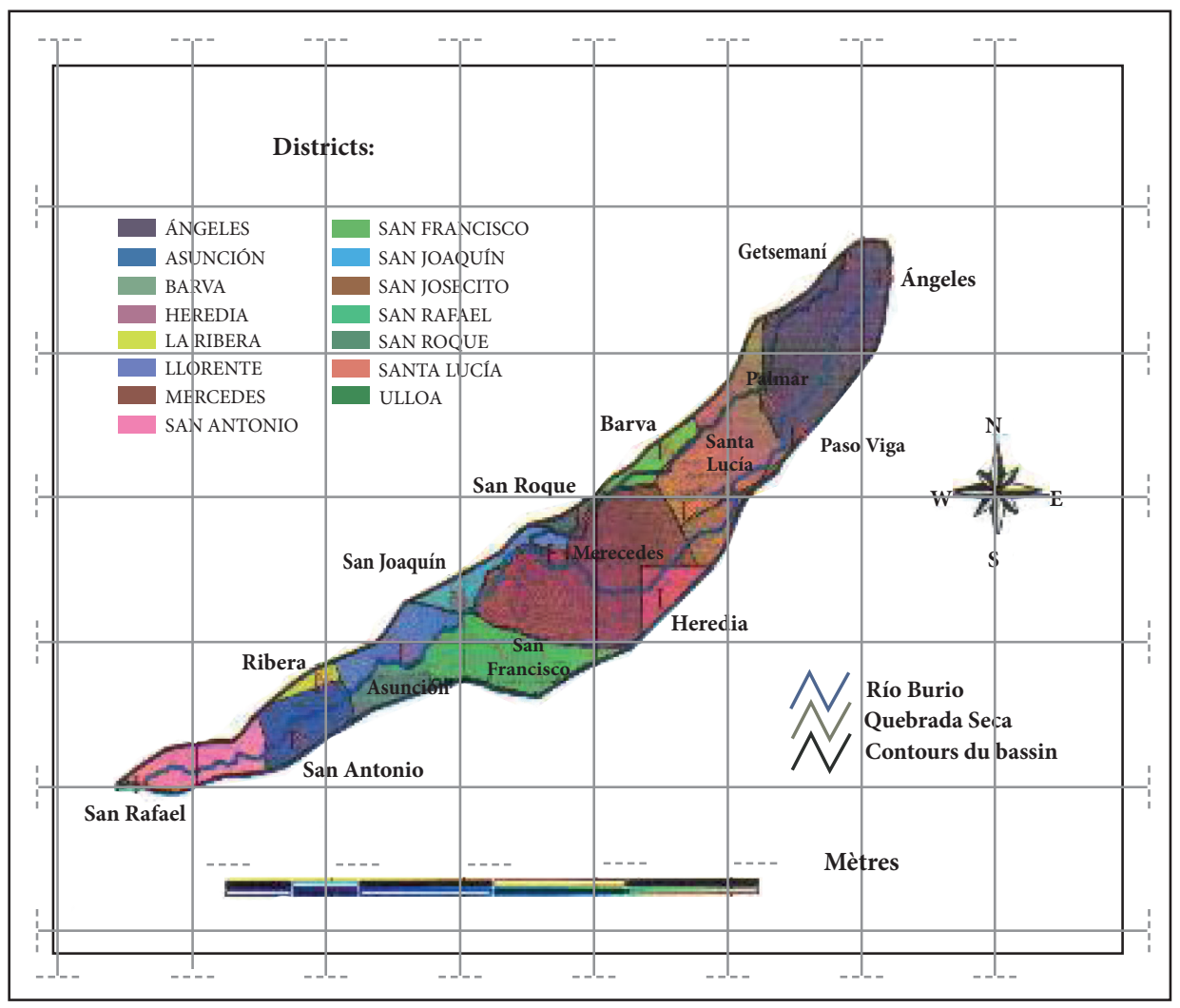

Figura 1. Microcuenca del río Burío-Quebrada Seca (Tisserant, 2008). 
La microcuenca tiene una longitud total de $17,5 \mathrm{~km}$ (Zamora, 2002), presenta una precipitación anual de $2042 \mathrm{~mm}^{3}$ y una temperatura promedio de $24,8^{\circ} \mathrm{C}$. Su altitud mínima es de 892 m.s.n.m. y la máxima de 1560 m.s.n.m. El clima es tropical lluvioso con estación seca, la cual se extiende desde abril hasta mediados de mayo. Presenta tres zonas de vida: bosque muy húmedo montano bajo (bmh-MB), bosque muy húmedo premontano (bmh-P) y bosque húmedo premontano (bhP). Sus suelos se caracterizan por ser de tipo inceptisoles (muy fértiles) (Sánchez, 2003).

Se seleccionaron 6 zonas de muestreo, distribuidas a lo largo de la microcuenca: dos en la parte alta, dos en la media y dos en la baja. Específicamente, las zonas de muestreo se ubicaron en: Parte alta: 1-Los Ángeles de San Rafael de Heredia y 2-El Palmar de San Rafael de Heredia. Parte media: 3-Santa Lucía de Barva de Heredia y 4-Mercedes Norte de Heredia. Parte baja: 5-San Antonio de Belén de Heredia y 6-San Rafael de Alajuela.

Las zonas fueron escogidas tomando en cuenta inspecciones de campo y giras de reconocimiento, interpretación de imágenes satelitales disponibles para la zona (Misión Carta 14 2005, escala 1:50 000) y concordancia con sitios en los cuales se ha desarrollado el proyecto "Hacia la gestión integrada del recurso hídrico en la microcuenca del río Burío-Quebrada Seca”, ejecutado por el Laboratorio de Manejo del Recurso Hídrico de la Universidad Nacional (LAMRHI). Estos criterios permitieron enlazar y relacionar los resultados de la presente investigación con resultados de otras investigaciones desarrolladas en el marco de dicho proyecto.

\section{Aplicación de índices QBR y QBRm}

Para efectos de este estudio se tomó como base la metodología desarrollada por Munné, Solá y Prat (1998), Suárez et al. (2002) y Munné et al. (2003), la cual consiste en la aplicación del índice llamado "calidad de bosque de ribera" o QBR (por sus siglas en el idioma catalán). La aplicación del índice permite clasificar el bosque ribereño, según su calidad, en una de cinco categorías: calidad muy buena, calidad buena, calidad intermedia, calidad mala y calidad pésima.

La investigación se realizó entre noviembre del 2009 y febrero del 2010, y de mayo a octubre del 2010. Primero se aplicó el índice QBR en las seis zonas de muestreo y posteriormente se diseñó y aplicó el índice QBRm, el cual es una adaptación del índice QBR.

El índice QBR evalúa la calidad de la ribera, con 4 parámetros o apartados biológicos y físicos del cauce, cada uno con un valor máximo de 25 puntos, para un puntaje máximo final de 100. Los parámetros son: el grado de cobertura de la ribera, la estructura de la cobertura, la calidad de la cobertura y el grado de naturalidad del canal fluvial. El resultado de la suma total de los apartados permite conocer la calidad de la ribera según los siguientes rangos: muy buena ( $\geq 95$ puntos), buena (71-94 puntos), regular (51-70 puntos), mala (26-50 puntos) y muy mala ( $\leq 25$ puntos).

El algoritmo que relaciona las características medidas con el índice y la calidad de la ribera es que cada uno de los apartados está asociado a características medibles porcentualmente y a una condición positiva o negativa, dependiendo tanto del apartado como de las características que se evalúan.

A modo de ejemplo, el apartado que califica el grado de cobertura de la ribera puede oscilar entre 0 y 25 puntos según el porcentaje de la cobertura de la vegetación que se observa en el punto de muestreo (oscila de 0 a 100\%). La característica asociada a este apartado es la "conectividad" entre el bosque de ribera y el ecosistema forestal adyacente; esto, con el fin de sumar o restar puntos según el cumplimiento de una de 4 condiciones posibles: si la conectividad es total, si es superior al $50 \%$, si es entre $25 \%$ y $50 \%$ o si es inferior al $25 \%$. 
UNICIENCIA Vol. 31, No. 1, pp. 39-49. Enero-junio, 2017.

El patrón anterior se cumple con los otros tres apartados cuyos detalles y características se pueden revisar en Munné et al. (1998). El índice QBR contempla 4 apartados, cada uno con un valor máximo de 25 puntos, para un puntaje máximo final de 100.

El índice QBRm se basa en el QBR y la modificación más importante es la incorporación de dos apartados relacionados con aspectos sociales. Para incluir los parámetros sociales en el índice QBRm se realizaron entrevistas semi-estructuradas entre los meses de mayo y agosto del 2010 los fines de semana, específicamente, los días sábado entre las 8:00 a.m. y las 2:00 p.m. Se aplicaron 15 entrevistas por zona, para un total de 90 en toda la microcuenca. En las entrevistas se indagó con las personas acerca de los siguientes temas: características sociodemográficas de la población, sistemas de saneamiento ambiental de las viviendas, servicios públicos recibidos y percepción y conceptos de las personas entrevistadas sobre diferentes aspectos relacionados con el río. El primer QBRm diseñado incluyó cuatro apartados nuevos, sin embargo, luego de un proceso de validación, se determinó que algunos no se relacionaban directamente con la afectación positiva o negativa de la actividad antrópica sobre las riberas, por lo que se aplicó un análisis multivariado jerárquico (programa estadístico Stata 11) para establecer la eficacia del índice QBRm en la determinación de la calidad de la ribera en cada una de las zonas de muestreo. Con los resultados obtenidos, se decidió eliminar dos apartados y conservar los siguientes: zona de protección al río y uso de la microcuenca y sistemas de saneamiento ambiental. Con estos apartados se buscó evaluar la relación que podría existir entre las características de la población que habita la zona de estudio y la condición de la vegetación ribereña y, consecuentemente, el estado de los ecosistemas. Al incorporar dos apartados y para conservar el índice en una escala de 0 a $100 \%$, se modificaron los aportes relativos de cada parámetro. Para más detalles de la modificación al índice original revisar Araya y Fernández (2011).

\section{- Zona de protección al río y uso de la microcuenca}

Este criterio permitió valorar el cumplimiento del Artículo 33 de la Ley Forestal número 7575 (La Gaceta, 1996). Se consideró de suma importancia porque el cumplimiento de esta normativa está relacionado con la vulnerabilidad del cuerpo de agua a la contaminación y los peligros potenciales para habitantes de las riberas. Fue importante efectuar un monitoreo de las casas que incumplen con la distancia de la zona de protección, ya que esto permitiría identificar las medidas a tomar para reducir el riesgo por inundaciones y para la protección o recuperación de las riberas, tanto a nivel biológico (reforestación, planes específicos enfocados al mejoramiento de la calidad del agua y los factores a tomar en cuenta para su implementación), así como a nivel social y legal (personas en riesgo, cumplimiento de la ley, permisos de construcción en casos similares, entre otras).

Se consideró también que el desarrollo de actividades económicas ligadas al río (actividades turísticas, recreativas y aprovechamiento del paisaje) era evidencia de que el curso de agua y sus alrededores se encontraban en condiciones que favorecían su aprovechamiento. Es importante contar con ríos que brinden oportunidades a la población de desarrollar actividades económicas, esto disminuye la migración, el desempleo, mejora los ingresos económicos (turismo) y aumenta el valor económico de las áreas influenciadas (Schmidt et al., 2011). Además, el uso del río para actividades recreativas evidencia buenas condiciones tanto de paisaje como de calidad de los recursos, refleja la percepción de las personas hacia el río y el interés en su protección. 


\section{- Sistemas de saneamiento ambiental}

La relación entre la condición de cursos de agua y de sus riberas, y las viviendas ubicadas en las cercanías o dentro de las zonas de protección, puede establecerse reconociendo los sistemas de tratamiento de aguas negras en la zona de estudio. Los estudios muestran que la baja cobertura de sistemas de alcantarillado en la provincia de Heredia hace que las aguas residuales sean vertidas en muchos casos en el alcantarillado pluvial o directamente en los ríos (Cañas, Guevara y Fonseca, 2008; Programa Estado de La Nación, 2010). Esta situación afecta todo el territorio nacional (Programa Estado de La Nación, 2010), a pesar de existir una obligación legal de tener y mantener un adecuado sistema de evacuación de aguas negras, así como una obligación de proteger los cuerpos de agua según la Ley General de la Salud (5395), Capítulo III (Obligaciones y restricciones para la evacuación de aguas servidas y negras) Artículos 275, 285, 287 y 288 y Capítulo VI (Deberes y restricciones relativos a las urbanizaciones y salubridad de las viviendas) Artículos 313, inciso7b y 314. La importancia de esta relación se resolvió en el índice QBRm con la asignación de un puntaje alto a este criterio.

La falta de un adecuado sistema de evacuación de las aguas pluviales hacia el alcantarillado público en las zonas urbanas de Heredia también es una de las principales causas de contaminación (mezcla con las aguas negras) y de inundación principalmente en la época lluviosa (Cañas et al. 2008). Se consideró importante evidenciar esta relación en el índice QBRm, ya que un adecuado sistema de evacuación de las aguas pluviales contribuye a reducir el problema de inundaciones, siempre y cuando se mantenga en buen estado el sistema de alcantarillado y la condición de las riberas.

Para determinar si existía una diferencia significativa entre los resultados obtenidos mediante la aplicación del índice QBR y el QBRm, se aplicó una prueba Chi cuadrado (niveles de confianza 90\% y 95\%), con el programa estadístico Stata 11.

\section{Resultados}

Mediante la aplicación del índice QBR se obtuvo una calidad “muy buena” para las zonas de muestreo 1 y 2 (95 puntos). La calidad de las zonas 3 y 4 fue "mala", al obtenerse puntajes de 40 y 45, respectivamente. Finalmente, la calidad en las zonas 5 y 6 fue "muy mala", con 15 puntos para la zona 5 y 10 puntos para la zona 6 .

El índice QBRm arrojó un puntaje de 80 para la zona de muestreo 1, lo cual la colocó en la categoría de calidad "buena"; la zona 2 también fue clasificada de calidad "buena" con un puntaje total de 73. Las zonas 3 y 4 obtuvieron una clasificación de "mala" calidad con un puntaje total de 33 y las zonas de muestreo 5 y 6 se caracterizaron como de "muy mala" calidad (23 puntos) y de "mala" calidad (27 puntos), respectivamente.

Al realizar una comparación entre los resultados obtenidos mediante la aplicación de los dos índices, se observó una tendencia hacia la disminución de la calidad de la ribera conforme se descendió en la microcuenca. Para las zonas de muestreo 1, 2, 3 y 4, los puntajes de calidad obtenidos con el índice QBRm fueron menores que los obtenidos con el índice QBR. Con respecto a las zonas 5 y 6, la puntuación asignada por índice QBRm fue mayor que la obtenida con el índice QBR (Tabla 1). 
UNICIENCIA Vol. 31, No. 1, pp. 39-49. Enero-junio, 2017.

Tabla 1

Porcentaje y calidad asignados por los indices QBR y QBRm en las seis zonas de muestreo a lo largo de la microcuenca del río Burío-Quebrada Seca.

\begin{tabular}{clclc}
\hline \multirow{2}{*}{ Zona de muestreo } & \multicolumn{2}{c}{ Índice QBR } & \multicolumn{2}{c}{ Índice QBRm } \\
\cline { 2 - 5 } & Categoría & Puntaje & Categoría & Puntaje \\
\hline 1 & Muy buena & 95 & Buena & 80 \\
2 & Muy buena & 95 & Buena & 73 \\
3 & Mala & 40 & Mala & 33 \\
4 & Mala & 45 & Mala & 33 \\
5 & Muy mala & 15 & Muy mala & 23 \\
6 & Muy mala & 10 & Mala & 27 \\
\hline
\end{tabular}

*Clasificación: $\geq 95$ Muy buena, 71-94 Buena, 51-70 Regular/Intermedia, 26-50 Mala, $\leq 25$ Muy mala.

\section{Discusión}

La comparación entre los resultados obtenidos con ambos índices mediante la prueba Chi cuadrado (p: 0.092) con un 95\% de confianza determinó que no existe una diferencia estadísticamente significativa entre la calidad de las zonas muestreadas y, por ende, entre la utilización del índice QBR y QBRm.

El QBRm demostró que, al incluir parámetros sociales, el puntaje final cambia con respecto al QBR y se apega más a la realidad observada en cada zona de muestreo, sin embargo, los resultados de la comparación no fueron estadísticamente significativos por lo que no se puede considerar como prioritario para utilizarlo sobre el QBR, ya que es necesario que se realice un análisis más profundo y más pruebas en otras microcuencas para determinar su efectividad.

Los resultados obtenidos conducen a dos opciones para la utilización de los índices QBR y QBRm. La primera sugiere que se debe mantener la utilización del índice QBR, respetando sus apartados específicos referentes estrictamente a la calidad de la vegetación y omitiendo las variables de tipo social, algunas de ellas difíciles de evaluar, especialmente por la necesidad de aplicar entrevistas u otra metodología con la cual se obtengan resultados de carácter social, porque no siempre las personas entrevistadas están anuentes a participar y por el tiempo que se requiere para la aplicación de estas.

En contraposición a lo anteriormente expuesto, el uso del QBRm tiene la ventaja de introducir a la población en la temática del manejo integrado de cuencas, actividad que permite que los sujetos habitantes de una unidad hidrográfica, al estar directamente implicados con la problemática de su entorno, cuenten con la oportunidad de dar a conocer sus conocimientos y experiencias, así como de participar en la gestión de este mismo. También, es un índice que brinda información útil para las autoridades gubernamentales municipales acerca del cumplimiento de las leyes relacionadas con la protección de las riberas, así como el acceso que tienen las personas a servicios básicos y la 
calidad de estos. A pesar de que aplicar las entrevistas puede ser difícil por el tiempo requerido, con una buena organización comunal se puede facilitar la entrega de los formularios con las preguntas y la aplicación final. De esta manera, el índice QBRm se convierte en una herramienta que recolecta datos que pueden ser usados para complementar el componente estrictamente biológico característico del índice QBR, el cual también podría ser analizado para adecuarlo a las características físicas de los ríos costarricenses y, de esta manera, poder evaluar la calidad de la zona de muestreo o de la microcuenca en estudio desde una perspectiva holística.

Hay que hacer hincapié en que el índice QBRm es una herramienta incipiente y mejorable con análisis de parámetros alternativos que se puedan incluir, buscando apartados que relacionen la parte biológica con las opiniones de las personas de las comunidades y realizando las pruebas estadísticas necesarias para fortalecerlo y ponerlo en práctica en diferentes microcuencas del país. Con base en los resultados obtenidos, se puede considerar la posibilidad de validarlo y aplicarlo en futuras investigaciones y considerarlo como una oportunidad para analizar la calidad de las riberas en las microcuencas del país.

\section{Referencias}

Aguilella, A., Riera, J., Gómez-Serrano, M. A., Mayoral, O., Moreyra, E., y Botànic, J. (2005). Evaluación del estado ecológico de los ríos de la cuenca hidrográfica del Júcar mediante el uso del índice QBR. Universitat de València. Valencia, España.

Araya, F. y Fernández, A. (2011). Análisis del estado de la vegetación ribereña de la microcuenca del río burío-quebrada seca, heredia, costa rica, mediante la aplicación de los índices calidad del bosque de riberas ( $q b r)$ y calidad del bosque de riberas modificado (QBRm) (Tesis de licenciatura inédita). Universidad Nacional, Costa Rica.

Burt, T. P., Pinay, G., Matheson, F. E., Haycock, N. E., Butturini, A., Clement, J. C., Danielescu, S., Dowrick, D. J., Hefting, M. M., Hillbricht-Ilkowska, A. y Maitre, V. (2002). Water table fluctuations in the riparian zone: comparative results from a pan-European experiment. Journal of Hydrology, 265, 129-148. http://dx.doi.org/10.1016/S0022-1694(02)00102-6

Cañas, D., Guevara, I. y Fonseca, J. (2008). Diagnósticos sobre seguridad ciudadana en diez cantones de Costa Rica ( $1^{a}$ ed.). Heredia, San José, Costa Rica: Programa de las Naciones Unidas para el Desarrollo.

Carlyle, G. C. y Hill, A. R. (2001). Groundwater phosphate dinamics in a river riparian zone: effects of hydrologic flowtpaths, lithology and redox chemistry. Journal of Hydrology, 247, 151-168. http:// dx.doi.org/10.1016/S0022-1694(01)00375-4

Ceccon, E. (2003). Los bosques ribereños y la restauración y conservación de las cuencas hidrográficas. Ciencias, 72, 46-53.

Coto, J. M., Benavides, A. C. y Salgado, V. (2008). Hacia la gestión integrada del recurso hídrico en la microcuenca del río Burío- Quebrada Seca. Laboratorio de Manejo del Recurso Hídrico, Universidad Nacional. Heredia, Costa Rica.

Dhondt, K., Boeckx, P., Verdehoest, N., Hofman, G. y Van Cleemput, O. (2006). Assessment of temporal and spatial variation of nitrate removal in riparian zones. Environmental Monitoring and Assessment, 116, 197-215. http://dx.doi.org/10.1007/s10661-006-7403-1

Díaz, W. y Rosales, J. (2006). Análisis florístico y descripción de la vegetación inundable de Várzeas Orinoquenses en el bajo del río Orinoco, Venezuela. Acta Botánica Venezolana, 29(1): 39-68.

Fernández, L., Rau, J. y Arriagada, A. (2009). Calidad de la vegetación ribereña del río Maullín (41 28'S; 72 59'O) utilizando el índice QBR. Gayana. Botánica, 66(2), 269-278. http://dx.doi.org/10.4067/ S0717-66432009000200011 
UNICIENCIA Vol. 31, No. 1, pp. 39-49. Enero-junio, 2017.

ISSN Electrónico: 2215-3470

URL: www.revistas.una.ac.cr/uniciencia

Email: revistauniciencia@una.cr

González, M. y García, D. (1998). Restauración de ríos y riberas. Madrid, España: Fundación Conde del Valle de Salazar y Ediciones Mundi-Prensa.

Granados, D., Hernández, M. A. y López, G. F. (2006). Ecología de las zonas ribereñas. Revista Chapingo, 12(1), 55-69.

Gregory, S., Frederick, S., McKee, W. A. y Cummins, K. W. (1991). An ecosystem perspective of riparian zones. Focus on links between land and water. BioScience, 41(8): 540-550. http://dx.doi. org/10.2307/1311607

Gutiérrez, M. R. V. A. y Alonso, M. L. S. (2000). Aplicación del índice de calidad del bosque de ribera, QBR (Munné et al., 1998) a los cauces fluviales de la cuenca del río Segura. Tecnología del agua, 201, 33-45.

Huggenberger, P., Hoehn, E., Breschta, R.y Woessner,W.(1998). Abioticaspect of channels and foodplainsin riparian ecology. Freshwater Biology, 40, 407-425. http://dx.doi.org/10.1046/j.1365-2427.1998.00371.x

La Gaceta. (1996). Diario Oficial. Boletín N. ${ }^{\circ} 72$. San José, Costa Rica: Imprenta Nacional.

Munné, A., Solá, C. y Prat, N. (1998). QBR: Un índice rápido para la evaluación de la calidad de los ecosistemas de ribera. Tecnología del Agua, 175, 20-37.

Munné, A., Prat, N., Solá, C., Bonada, N. y Rieradevall. M. (2003). A simple field method for assessing the ecological quality of riparian habitat in rivers and streams: QBR index. Habitat. Aquatic Conservation. Marine and Freshwter Ecosistems, 13, 147-163. http://dx.doi.org/10.1002/aqc.529

Oviedo, E. (2007, 2 de diciembre). Heredianos sufren por río que contaminaron por años. La Nación. Recuperado de http://wvw.nacion.com/ln ee/2007/diciembre/02/pais1322627.html

Palma, A., Figueroa, R., y Ruiz, V. H. (2009). Evaluación de ribera y hábitat fluvial a través de los índices QBR e IHF. Gayana (Concepción), 73(1): 57-63. http://dx.doi.org/10.4067/S0717-65382009000100009

Pinay, G. y Decamps, H. (1988). The role of riparian woods in regulating nitrogen fluxes between the alluvian aquifer and surface water. A conceptual model. Regulated Rivers, Research and Management, 2, 507-516. http://dx.doi.org/10.1002/rrr.3450020404

Programa Estado de la Nación. (2010). Decimosexto Informe Estado de la Nación en Desarrollo Humano Sostenible (Capítulo 1, Sinopsis). San José, Costa Rica: Autor.

Rodríguez, J. (2006). Análisis socioecológico de la vegetación ribereña en la cuenca baja del río Ayuquila. Elementos para su rehabilitación. (Tesis de licenciatura inédita). Centro Universitario de la Costa del Sur. Universidad de Guadalajara. Guadalajara, México.

Rodríguez-Téllez, E., Domínguez-Calleros, P. A., Pompa-García, M., Quiroz-Arratia, J. A. y M. E. Pérez López. (2012). Calidad del bosque de ribera del río El Tunal, Durango, México; mediantela aplicación del índice QBR. Gayana. Botánica, 69(1), 147-151. http://dx.doi.org/10.4067/S0717-66432012000100014

Sánchez, V. (2003). Gestión ambiental participativa de microcuencas. Fundamentos y aplicación: El caso de la quebrada Salitral Costa Rica. Heredia, Costa Rica: EUNA

Schmidt, G., Arribas, T., Cavallé, A., López, A., Molina, J. R., Palacios, E., Morillo, R., Del Valle, M. y A. Ballester. (2011). Procesos de participación pública en la restauración de ríos. Manuales de desarrollo sostenible 10. Recuperación de Riberas. España: Fundación Banco Santander.

Sorensen, M., Barzetti, V., Keipi, K y J. Williams. (1998). Manejo de las áreas verdes urbanas. Washington, Estados Unidos.

Suárez, M. L., Vidal-Abarca, M. R., Sánchez-Montoya, M.; Alba-Tercedor, J., Álvarez, M., Avilés, J., Bonada, N., Casas, J., Jáimez-Cuéllar, P., Munné, A., Pardo, I., Prat, N., Rieradevall, M., Salinas, M. J. Toro, M. y S. Vivas. (2002). Las riberas de los ríos mediterráneos y su calidad: El uso del índice QBR. Limnética, 21(3-4), 135-148. 
ISSN Electrónico: 2215-3470

DOI: http://dx.doi.org/10.15359/ru.31-1.5
UNICIENCIA Vol. 31, No. 1, pp. 39-49. Enero-junio, 2017. URL: www.revistas.una.ac.cr/uniciencia Email: revistauniciencia@una.cr

Timoney, K.P., Peterson, G. y Wein, R. (1997). Vegetation development of boreal riparian plant communities after flooding, fire and logging, Peace river, Canada. Forest Ecology and Management, 93: 101-120. http://dx.doi.org/10.1016/S0378-1127(96)03929-1

Tisserant, A. (2008). Analyse des problèmes liés à l'érosion des berges du Río Burío - Quebrada Seca (Province de Heredia): Valorisation des données existantes et acquisition in situ. (Práctica profesional). Universidad Nacional Autónoma, Costa Rica.

Zamora, R. A. (2002). Diagnóstico físico-natural y características del uso de la tierra de la microcuenca del río Burío y su tributaria quebrada Seca Heredia, Costa Rica (Tesis inédita de licenciatura). Universidad Nacional Autónoma, Costa Rica.

\section{(2) $(\mathcal{Q} \Theta \Theta$}

Modificación del Índice de Calidad de Riberas: inclusión del componente social en la evaluación de la calidad ribereña de la microcuenca del río Burío-Quebrada Seca (Fabián Araya-Yannarella y otros) por Revista Uniciencia se encuentra bajo una Licencia CreativeCommons Atribución-NoComercial-SinDerivadas 3.0 Unported. 